\title{
latrogenic Genitourinary Fistula: A Rising Trend
}

\author{
TAUFIQUA HUSSAIN ${ }^{1}$, AFRINA BEGUM ${ }^{2}$, NASRIN AKHTER ${ }^{3}$, TABASSUM GHANI ${ }^{4}$, \\ NOORJAHAN ${ }^{5}$, DINALAYLA HOSSAIN ${ }^{6}$, GUL-A-ANAR ${ }^{7}$
}

\begin{abstract}
Background:Obstetric fistula (OF) is not very uncommon in our country. But recent issue of increase in iatrogenic fistula (IF) creates a field of interest.

Objective:To find out the frequency and the cause of iatrogenic genitourinary fistula admitted in National Fistula Centre (NFC), Dhaka.

Methods:This prospective study was conducted in NFC, Dhaka from January, 2010 to December, 2012. All cases admitted during the study period were included in this study. All patients were evaluated through detailed history, physical examination, relevant investigations and examination under anaesthesia (EUA). Repair was done through abdominal or vaginal route based on the findings. Results of repair were noted and analyzed.

Results:On an average more than $20 \%$ cases of genitourinary fistula (GUF) were due to iatrogenic causes. Among them about $90 \%$ cases had history of hysterectomy as an offending procedure. A rising trend of iatrogenic fistula is observed with $19.04 \%$ in 2010 , $15.68 \%$ in 2011 and $30 \%$ in 2012.

Conclusion:This study shows that iatrogenic injuries in women under 40 years of age form major share in the aetiology of GUF. latrogenic fistula are increasing day by day. It is the time to check the experience of surgeons doing the gynecological and obstetrical surgeries in a developing country like ours.
\end{abstract}

Key Words: Genitourinary fistula, latrogenic fistula, Hysterectomy.

\section{Introduction}

Genitourinary fistula (GUF) is an abnormal communication between genital and urinary tract. Fistula is as old as mankind but its documentation is much younger than that. There were however earlier references to fistula, even as early as $1550 \mathrm{BC}$. ${ }^{1}$ This complication has been recognized when the mummy of queen Henhenit dating back to 2000 BC was examined in $1942 .{ }^{2}$ It is still a common problem in developing world, as $84 \%-97 \%$ of cases occur in these countries. ${ }^{3}$ In developing countries the major cause is obstructed labour while in developed countries $90 \%$ are caused by gynecological producers. ${ }^{4,5}$

In Bangladesh obstructed labour is still considered as the major cause of GUF. It is responsible in $80-90 \%$ of cases. ${ }^{6} \mathrm{WHO}$ estimates the prevalence of obstetric fistula $0.3 \%$ of all deliveries. ${ }^{7}$ In recent years it is observed that there is a change in trend in the aetiology of fistula. latrogenic injury is gradually increasing as a cause of GUF.

An IF is an abnormal communication between the bladder or ureter with the uterus / cervix / vagina, resulting from surgical procedure. ${ }^{8}$ The type of fistula which occur as a result of iatrogenic injury may be vesicovaginal,vesico-uterine, vesico-cervical and ureterovaginal. The reason for increasing trend of IF may be due to marked improvement in skilled birth attendance, may be due to timely referral, may be due to increasing facility for emergency caesarean section at periphery. But side by side gynecological operative procedures

1. Associate Professor, Obs\&Gynae, Dhaka Medical College.

2. Associate Professor, Obs\&Gynae, Dhaka Medical College.

3. Assisstant Professor, Obs\&Gynae, Dhaka Medical College.

4. AssisstantProfessor, Obs\&Gynae, Dhaka Medical College.

5. Assisstant Professor, Obs\&Gynae, Dhaka Medical College.

6. Registrar, Obs\&Gynae, Dhaka Medical College Hospital.

7. Senior consultant, TangailSadar Hospital.

Address of Correspondence: Dr. Taufiqua Hussain, Associate Professor(Gynae), Dhaka Medical College, email: taufiqua.1959@gmail.com, mobile: 01711423203. 
are performed in the periphery by doctors not trained sufficiently in this specialty. Therefore, this study was conducted to find out the changing trend as well as the frequency of iatrogenic injuries as the aetiology of IF of all the patients admitted for treatment in NFC, Dhaka.

\section{Methods}

This is a prospective cross sectional observational study carried out in NFC, Dhaka, from January, 2010 to December, 2012. All patients complaining of urinary incontinence due to GUF were included in the study. Patients having stress incontinence and recto-vaginal fistula were excluded from the study.

A detailed history was taken to record different variables like age, parity, time interval of appearing symptoms after surgery or delivery, duration of leaking, type of fistula, cause of fistula and whether any previous attempts of repair. After informed verbal consent the patient was subjected to thorough physical examination, along with routine investigation, Examination Under Anesthesia (EUA) was performed to assess the number, site, size of fistula. Ultrasonography, cystoscopy and intravenous urography (IVU) were done in some selected cases, specially where confusion occured and to confirm the diagnosis. On the basis of findings, route of operation either abdominal or vaginal was planned. The cases were then operated upon by the gynecologists and if necessary urologists were also involved. Postoperatively cases were nursed with close supervision. Parenteral antibiotics were given postoperatively. Adequate hydration was maintained and special care was taken for continuous drainage of urine for 21 days. Routine urine examination was done to exclude any infection. In case of any infection, culture and sensitivity test of urine was done and specific antibiotics were given. Culture and sensitivity test of high vaginal swab was done as routine investigation after removal of vaginal pack 48 hours after operation, if given. Success rate was evaluated as symptom free state for 24-48 hours after removal of catheter. Data was analyzed manually. Simple descriptive statistics were calculated.Age was presented as frequency distribution table. Frequency and percentage were calculated for certain variables like type of fistula and cause of fistula.

\section{Results}

During the study period total patient admitted in NFC were 570. Out of them 36 patients were excluded due to reason other than GUF. They were the cases of stress incontinence, fecal incontinence,bladder stone,vaginal stenosis etc. Remaining 534 patients were operated for GUF. Age distribution of patients is given in table-I. Total number of IF operated were 108. The number of iatrogenic cases following hysterectomy were 94. Eighty six (86) cases after hysterectomy for gynecological indication and eight (8) cases after caesarean hysterectomy for indication other than obstructed labour. Number of uretero-vaginal fistula (UVF) were 15. Following gynecological hysterectomy number was 12 and 3 after emergency caesarean hysterectomy. There were 8 cases of vesico uterine fistula (VUF), 2 following caesarean section for obstructed labor and 6 cases following caesarean section for indication other than obstructed labor. Rest 85 cases of IF is vesico-vaginal fistula (VVF), majority of them following routine hysterectomy. Among the OF cases 290 delivered vaginally, 128 following caesarean section for obstructed labour, only 8 cases for caesarean hysterectomy. Causes of caesarean hysterectomy were postpartum haemorrhage and ruptured uterus. No alive baby was delivered in any case of OF.

Table-I

Distribution of patients according age group

\begin{tabular}{lcc}
\hline Age & OF & IF \\
\hline$<20$ years & $50(11.73 \%)$ & $4(3.70 \%)$ \\
$20-40$ years & $275(64.55 \%)$ & $70(64.81 \%)$ \\
$>$ 40 years & $101(23.70 \%)$ & $34(31.48 \%)$ \\
\hline Total & $426(100 \%)$ & $108(100 \%)$ \\
\hline
\end{tabular}

Table-II

Cause of fistula

\begin{tabular}{llccc}
\hline Cause & Procedure & No. of patients & Total & Percentage \\
\hline IF & Hysterectomy (Gynecological) & 86 & & \\
& Hysterectomy (Caeserean) & 8 & & \\
& Caesarean section (other than obstructed labour) & 9 & 108 & $20.22 \%$ \\
& Caesarean section (for obstructed labour) & 5 & & \\
OF & Post delivery & 290 & & \\
& Caesarean section for obstructed labor & 128 & 426 & $79.77 \%$ \\
& Caesarean hysterectomy & 8 & & \\
\hline
\end{tabular}


The causes of fistula both iatrogenicand obstetric are shown in table-II. The proportion of IF versus OFwas calculated year wise, which is shown in fig1. Proportion of IF is $19.04 \%, 15.68 \%$ and $30 \%$ in the year 2010, 2011 and 2012 respectively.

$$
\text { OF } \square \text { IF }
$$

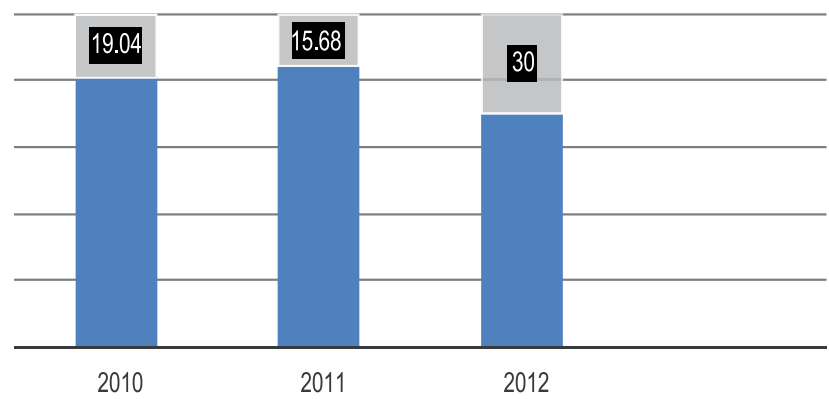

Fig.-1

All the cases operated to repair the fistula and to make the patient continent. Route of operation was chosen according to the need of the patient. Local repair of fistula was done in vaginal route in maximum cases. Abdominal route was for uretero-neocystostomy and some special cases where special involvement of urologist colleagues were needed. Success rate is more than $90 \%$ in IF whereas it is only $61 \%$ in OF cases. Success rate of fistula repair is shown in table -III.

Table-III

Success rate of fistula repair

\begin{tabular}{lcc}
\hline Type & Successful & Failed \\
\hline IF & $98(90.74 \%)$ & $10(9.25 \%)$ \\
OF & $260(61.03 \%)$ & $166(38.96 \%)$ \\
\hline
\end{tabular}

\section{Discussion}

GUF still ranks as one of the major gynecological problems in many developing countries like ours. Its impact affects the social life of women.

Uncontrolled leakage of urine into the vagina or incontinence along with odour and discomfort causes serious social problems resulting in multifaceted problems for women. ${ }^{9}$

In this study majority of patients were below the age of 40 . The problem is that majority of the cases occurred in younger age group and if not treated the women would have to spend a long part of their life with this affliction, creating social and psychological problems in addition to medical issues.

Different studies in Bangladesh has labled obstructed labour as the most common aetiological factor for OF,, .10 .11 while in the developed countries, gynecological procedures are considered to be responsible for most of the GUF. Among the GUF $82 \%$ were due to gynecological surgery for benign diseases ${ }^{12}$. Surgical injury to the lower urinary tract most commonly occurs in the setting of hysterectomy ${ }^{13}$. Most authors quote on incidence of VVF after total abdominal hysterectomy (TAH) to be $0.5-2 \%$, others suggest only a $0.05 \%$ injury rate either the bladder or ureter ${ }^{14}$. However in this series about $87 \%$ of the cases of IF occurred following hysterectomy. This finding correlates with other study 5.15 .

In most cases indication for hysterectomy was gynecological and specially for benign disease ${ }^{12}$.

During last several years'different studies in tertiary care hospital in Bangladesh showed negligible proportion of IF, That is not more than $2 \%-4 \% 6,10$. In a study in Niger from June 11- June 14 the proportion of IF was $9.9 \%^{16}$. In this study the proportion of IF on an average in more than $20 \%$, which in more than the previous 3-4 years record of the same centre as well as more than the mentioned studies. There is remarkable increase in 2012, which is $30 \%$ (fig: 1 ).

Majority of the patients who came to NFC had been operated in private clinics in the periphery by surgeon inexperienced in this field and lacked proper training also. In a study in tertiary care hospital in Bangladesh showed $90 \%$ of the gynecological surgery was done by non-gynecologist and nonspecialist ${ }^{15}$.

Successful repair of fistula depends on many factors. Patients may present with other associated problems which complicate and interfere in the successful repair ${ }^{17}$. But IF is comparatively free from associated problems which is very common in OF, specially following obstructed labour. In our study success rate of repair of IF in more than $98 \%$ (table-iv), which is comparable with other study ${ }^{15}$.

This study is limited and may be biased. OF recognized the consequence of health system failure and the inability of the world's poorest and most marginalized women to access emergency obstetric care. So reduction of OFcases indicates the 
improvement of health systems, no doubt. But prevention of IF is an urgent matter that needs to be addressed, specially in developing countries because most of IF caused due to some gynecological procedures performed in the periphery by the person without adequate knowledge and skill. Providers performing obstetric and gynaecological surgery must have the appropriate competencies. Training combined with monitoring and ongoing supervision is essential.

\section{Conclusion}

This study shows a rising trend in iatrogenic GUF in the last several years of the patients admitted in NFC. As most of the patients came from periphery, it is an indication that gynecological surgery is not being performed by qualified surgeon. There should be a strong audit system for major surgical procedures,

\section{References}

1. Akhter S. Principles \& Practices in management of female genital tract fistula. April 2008; 3.

2. Spurlock J. Vesico vaginal fistula (Inter net). [Updated 2009, Oct.1] Available from: http:// emedicine.medscape.com/article/267943overview.

3. Raut V. Bhattacharya M. Vesical fistulae: An experience from a developing country. J. Post grad Med 1993; 39: 20-1

4. Smith GL, Williams G. Vesicovaginal fistulae. BJU Int. 1999; 83: 564-70.

5. Tancer ML. Observations on prevention and management of vesicovaginal fistula after total hysterectomy.SurgGynecolobstet 1992; 175: 501-6.

6. Begum SN. Genitourinary Fistula- Experience in a Peripheral Hospital of Bangladesh. J Bangladesh CollPhysSurg 2011; 29: 207-212.

7. World Health Organization, 2004. Global action for skilled attendants for pregnant women. WHO/
RHR/02.17. 2004, www:who.int/reproductive health/publications.

8. Thomas J.I.P Raassen, Carrie J. Ngongo, Marietta M, Mahendeka. latrogenic genitourinary fistula: an 18-year retrospective review of 805 injuries. Int.Urogynecol J. 2014 October 15; 25: 1707.

9. Rashid Y, Majeed T, Majeed N, Shahzad N, Tayyab S. Jaffri H. latrogenic vesicovaginal Fistula. J Coll Physicians Surg Pak 2010, 20 (7): 436-38.

10. Hussain T. genitourinary fistula- A clinical study of eighty cases (Dissertation), Dhaka. Bangladesh College of physicians and surgeons, 1994.

11. Islam F. Genital fistula- A clinical study (Dissertation) Bangladesh college of physicians and surgeons, 1990.

12. Lee RA, Symmods RE, William TJ. Current status of genitourinary fistula.ObstetGynecol 1988; 72: 313-9.

13. Michael Stamatakos, Constantinasargedi and KonstantinosKontzoglou. Vesicovaginal Fistula: Diagnosis and Management. Indian J Surg, 2014 April; 76 (2): 131-36

14. American College of obstetrician \&Gynaecologist.Genitourinary fistula.ACOG Technical Bulletin 1985; 83: 1-6.

15. S. Nahar, MK Sarkar, KP Das, NAkter. latrogenic Genitourinary Fistula: A survey in Khulna Medical College. J. Bangladesh CollPhySurg 2005; 23, 68-71.

16. Go Sanda, BA Diagne, SM Gueye, A Harisson, ASoumana.Contribution of latrogenic Cause to Female Genital Fistula Burden in a New Classification System. Global Maternal Newborn Conference, October, 2015.

17. Begum HA. Outcome of surgical repair of vesicovaginal fistula. J Dhaka Medical Col, 1996; 5(1), 16-20. 